\title{
Erratum to: Cerebral amyloid angiopathy in a young man with a history of traumatic brain injury: a case report and review of the literature
}

\author{
Yoichi Nakayama $^{1}$ - Yohei Mineharu ${ }^{1} \cdot$ Yoshiki Arakawa $^{1} \cdot$ Sei Nishida $^{1}$. \\ Hirofumi Tsuji $^{1}$ - Hidehiko Miyake ${ }^{2}$. Maki Yamaguchi ${ }^{3}$ - Sachiko Minamiguchi ${ }^{3}$. \\ Yasushi Takagi $^{1} \cdot$ Susumu Miyamoto $^{1}$
}

Published online: 3 December 2016

(C) Springer-Verlag Wien 2016

Erratum to: Acta Neurochir

DOI 10.1007/s00701-016-3004-0

The original version unfortunately incorrectly spelt the name of Yoshiki Arakawa and is now corrected in this article.

The online version of the original article can be found at http:10.1007 /s00701-016-3004-0.

\footnotetext{
Yohei Mineharu

mineharu@kuhp.kyoto-u.ac.jp

1 Department of Neurosurgery, Kyoto University Graduate School of Medicine, 54 Shogoin Kawahara-cho, Sakyo, Kyoto 606-8507, Japan

2 Clinical Genetics Unit, Kyoto University Graduate School of Medicine, 54 Shogoin Kawahara-cho, Sakyo, Kyoto 606-8507, Japan

3 Department of Diagnostic Pathology, Kyoto University Graduate School of Medicine, 54 Shogoin Kawahara-cho, Sakyo,

Kyoto 606-8507, Japan
} 